\title{
谱图小波阈值降噪及其在滚刀主轴振动信号 分析中的应用*
}

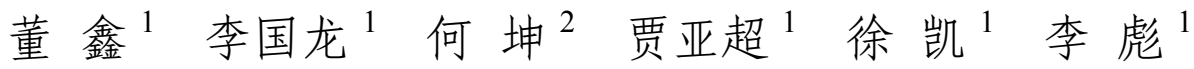 \\ (1. 重庆大学机械传动国家重点实验室 重庆 400030; \\ 2. 重庆工商大学机械工程学院 重庆 400067)
}

\begin{abstract}
摘要: 通过分析谱图小波变换在平面图像、三维实体分析中的应用, 提出一种用于一维数字信号分析的谱图小波阈值降噪方 法。该方法将一维数字信号定义到路图上, 利用谱图小波变换将其分解成尺度系数和谱图小波系数, 对谱图小波系数进行阈 值过滤处理, 再进行谱图小波逆变换得到降噪信号。首先, 利用四种典型仿真信号进行降噪试验, 并分析不同分解层数对降 噪性能的影响; 接着, 将其与经典小波阈值降噪方法进行仿真对比; 最后, 采用该方法进行滚刀主轴振动信号降噪, 并与经 典小波阈值降噪方法对比。仿真及试验结果表明, 该方法实现了一维数字信号的快速非迭代降噪, 且降噪信号平滑度高、畸 变小, 优于经典小波阈值降噪方法。
\end{abstract}

关键词: 谱图小波变换; 阈值; 降噪; 滚刀主轴; 振动信号

中图分类号: TN911

\section{Spectral Graph Wavelet Threshold Denoising and Its Application to Vibration Signal Analysis for Hob Spindle}

\author{
DONG Xin ${ }^{1}$ LI Guolong ${ }^{1}$ HE Kun ${ }^{2}$ JIA Yachao ${ }^{1}$ XU Kai ${ }^{1}$ LI Biao ${ }^{1}$ \\ (1. State Key Laboratory of Mechanical Transmission, Chongqing University, Chongqing 400030; \\ 2. College of Mechanical Engineering, Chongqing Technology and Business University, Chongqing 400067)
}

\begin{abstract}
By analyzing the application of spectral graph wavelet transform in plane image and three-dimensional solid analysis, a spectral graph wavelet threshold denoising method is proposed for one-dimensional digital signal analysis. In this method, the one-dimensional digital signal is defined on the path graph, which is decomposed into scale coefficients and spectral graph wavelet coefficients by spectral graph wavelet transform, and the spectral graph wavelet coefficients are filtered by threshold. Thus the denoising signal is obtained by spectral graph wavelet inverse transform. Firstly, four typical simulation signals are used for denoising test, and the influence of different decomposition layers on denoising performance is analyzed. Then, the performance of the proposed method is compared with that of the classical wavelet threshold denoising method by simulation. Finally, the method is applied to the denoising experiment of hob spindle vibration signal, and compared with the classical wavelet threshold denoising method. Simulation and experimental results show that the proposed method can realize the fast non-iterative denoising of one-dimensional digital signal, and the denoising signal has high smoothness and small distortion. Also, it is obviously superior to the classical wavelet threshold denoising method.
\end{abstract}

Key words: spectral graph wavelet transform; threshold; denoising; hob spindle; vibration signal

\section{0 前言}

数字信号在工业制造智能化转型升级进程中起

\footnotetext{
* 国家自然科学基金资助项目(51875066)和重庆市基础研究与前沿探索 (cstc2018jcyjAX0578)资助项目。20190621 收到初稿, 20200330 收到 修改稿
}

着举足轻重的作用, 提取并分析数控机床的各种数

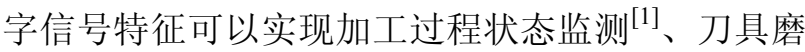
损识别 $^{[2-3]}$ 、故障诊断 ${ }^{[4]}$ 、误差补偿 ${ }^{[5]}$ 等智能化功能。 然而数字信号在采集与传输过程中易受到采集仪 器、周围环境及人为因素影响, 造成采集的实际信 号中掺杂部分噪声 ${ }^{[6]}$, 导致提取的信号特征难以准 确反映机床的运行状态。因此, 须在提取特征前对 
采集的数字信号进行降噪处理。

数字信号处理的常用方法是将数据从原始域映 射到其它域中进行分析 ${ }^{[7]}$, 其中映射方法包括离散 傅里叶变换(Discrete Fourier transform, DFT)、小波 变换(Wavelet transform, WT)、希尔伯特变换(Hilbert transform, HT)、变分模态分解(Variational mode decomposition, VMD)、经验模态分解 (Empirical mode decomposition, EMD)等。近年来发展起来的 谱图小波变换 ${ }^{[8]}$ (Spectral graph wavelet transform, SGWT)引起了学术界越来越多的关注, 其类似于定 义在加权图上信号的傅里叶变换, 结合了谱图理论 和经典小波变换的相关特性, 能够根据图信号的频 谱特性将其分解成不同频率区间的子带, 实现在谱 图域中分析图信号的特征。HAMMOND 等 ${ }^{[8]}$ 系统性 地研究了谱图小波变换理论及其相关性质, 并举例 说明了谱图小波变换在交通网络、计算机网络、脑 成像等方面的应用前景。然后, 越来越多的国内外 学者开始探索谱图小波变换的具体应用。在平面图 像应用方面, LEANDRO 等 ${ }^{[9]}$ 将谱图小波变换用于 构建星系形态图像的表征框架, 并以监督学习和非 监督学习方法验证了所提出框架的适用性。DONG 等 ${ }^{[10]}$ 基于谱图小波变换讨论了基于空间局域事件 的地理图移动模式问题, 可使用粗糙的聚合信息进 行移动模式的有效推断。OZDEMIR 等 ${ }^{[11]}$ 将图像转 换到图域, 利用谱图小波变换对图像进行分割, 实 现了图像在像素域的不连续性检测。BEHJAT 等 ${ }^{[12]}$ 基于谱图小波变换构建核磁共振脑成像, 提高了大 脑活动检测灵敏度。DEUTSCH 等 ${ }^{[13]}$ 提出了一种基 于谱图小波变换的光滑流形降噪方法, 其通过保留 总积累能量高于阈值 $E$ 的低频对应的所有尺度系数 和谱图小波系数, 丢弃其余高频对应的谱图小波系 数, 实现了二维图像的降噪。王林等 ${ }^{[14]}$ 提出了一种 基于谱图小波变换的图像压缩编码方法, 在高压缩 比下也能得到较好质量的重构图像。在三维实体应 用方面, MASOUMI 等 ${ }^{[15]}$ 运用谱图小波变换建立了 一个描述三维模型特征的框架, 能同时捕捉三维模 型的局部特征和全局特征, 实现了三维实体形状检 索。胡玲等 ${ }^{[16-17]}$ 将谱图小波变换用于三维模型几何 处理, 实现了模型的多分辨表示, 且表现出较高的 形状分辨能力和鲁棒性。

综上所述, 谱图小波变换在平面图像、三维实体 分析中已经取得了一些研究成果。本文在深入分析谱 图小波变换理论及其相关应用的基础上, 提出一种用 于一维数字信号分析的谱图小波阈值降噪(Spectral graph wavelet threshold denoising, SGWTD)方法, 并
采用经典仿真信号及滚刀主轴振动信号进行降噪试 验验证谱图小波阈值降噪方法的可行性和有效性。

\section{1 谱图小波变换基本理论}

谱图小波变换是基于谱图理论在图域上构建的 小波变换, 其通过设计一组低通和高通滤波器实现 图信号的多频带分析。

\section{1 经典小波变换基本概念}

经典小波变换由母小波 $\psi(t) \in L^{2}(R)$ 决定。引入 尺度因子 $s$ 和位移因子 $a$, 得到对应不同尺度和位 置的一簇小波, 可表示为

$$
\psi_{s, a}(t)=\frac{1}{\sqrt{s}} \psi\left(\frac{t-a}{s}\right)
$$

对于给定信号 $f(t)$, 尺度 $s$ 和位置 $a$ 处的小波 系数由 $f$ 与小波 $\psi_{s, a}$ 的内积得出, 即

$$
W_{f}(s, a)=\left\langle\psi_{s, a}, f\right\rangle=\frac{1}{\sqrt{s}} \int_{-\infty}^{+\infty} \psi^{*}\left(\frac{t-a}{s}\right) f(t) \mathrm{d} t
$$

\section{2 谱图理论基本概念}

谱图理论 ${ }^{[18]}$ 主要用于建立离散空间和连续空 间之间的联系, 并通过图的邻接矩阵、拉普拉斯矩 阵、拟拉普拉斯矩阵等研究矩阵的谱性质, 从而提 取图中包含的有用信息。

给定无向加权图 $G=(\boldsymbol{V}, \boldsymbol{E}, \omega)$, 其中 $\boldsymbol{V}$ 表示图 $G$ 的节点集 $(|\boldsymbol{V}|=N$ 表示节点数), $\boldsymbol{E}$ 表示图 $G$ 的边集 $(|\boldsymbol{E}|=M$ 表示边数); $\omega$ 对应边的权值, 常用 Gauss 权值函数定义权值

$$
\omega_{i j}=\exp \left(-\left\|x_{i}-x_{j}\right\|^{2} / 2 \sigma^{2}\right)
$$

式中, $\omega_{i j}$ 表示边 $e_{i j}=v_{i} v_{j}$ 的权值, $x_{i}$ 和 $x_{j}$ 分别为节 点 $i$ 和节点 $j$ 对应的函数值, $(\|\cdot\|)$ 表示节点间的欧 氏距离, $\sigma$ 表示热核的宽度。

图 $G$ 的邻接矩阵 $\boldsymbol{W}$ 定义为

$$
\boldsymbol{W}=\left(e_{i j}\right)= \begin{cases}\omega_{i j} & v_{i} \text { 与 } v_{j} \text { 相邻 } \\ 0 & \text { 其他 }\end{cases}
$$

图 $G$ 的度对角矩阵 $\boldsymbol{D}$ 定义为

$$
\begin{aligned}
\boldsymbol{D} & =\operatorname{diag}\left(d_{i}\right) \\
d_{i} & =\sum_{j=1, j \neq i}^{N} \omega_{i j}
\end{aligned}
$$

图 $G$ 的拉普拉斯矩阵 $\boldsymbol{L}$ 建立在邻接矩阵 $\boldsymbol{W}$ 的 基础上, 并包含节点的度信息, 定义为

$$
\boldsymbol{L}=\boldsymbol{D}-\boldsymbol{W}
$$

$\boldsymbol{L}$ 为实对称矩阵, 进行标准正交分解得到非负 的实拉普拉斯特征值 $\lambda_{l}$ 和正交拉普拉斯特征向量 $\boldsymbol{x}_{l}$, 满足 


$$
\boldsymbol{L} \boldsymbol{x}_{l}=\lambda_{l} \boldsymbol{x}_{l}, l=0,1, \cdots, N-1
$$

且 $\lambda_{l}$ 可排序为 $0=\lambda_{0}<\lambda_{1} \leqslant \lambda_{2} \leqslant \cdots \leqslant \lambda_{N-1}=\lambda_{\text {max }}$, 其 中 $\lambda_{\text {max }}$ 为拉普拉斯特征值上界。

路图是结构最简单的图, 可视其为节点与节点 通过边依次连接组成的序列, 如图 1 所示。

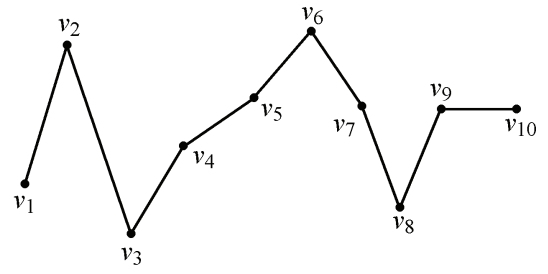

图 1 路图

图 1 中, $v_{1} 、 v_{2} 、 v_{3} 、 v_{4} 、 v_{5} 、 v_{6} 、 v_{7} 、 v_{8} 、$ $v_{9} 、 v_{10}$ 为节点, 节点数 $N=10 ; v_{1} v_{2} 、 v_{2} v_{3} 、 v_{3} v_{4}$ 、 $v_{4} v_{5} 、 v_{5} v_{6} 、 v_{6} v_{7} 、 v_{7} v_{8} 、 v_{8} v_{9} 、 v_{9} v_{10}$ 为连接相邻节 点的边, 边数 $M=9$ 。对于任意路图, 节点数 $N$ 与边 数 $M$ 关系为: $N=M+1$ 。

\section{3 谱图小波变换基本概念}

SGWT 由谱图小波核 $g$ 决定, 其通过谱图小波 算子 $T_{g}=g(\boldsymbol{L})$ 调制每个图的傅里叶模式作用于给 定的图信号 $f$ 。类比于经典小波变换的尺度变换, $T_{g}^{t}=g(t \boldsymbol{L})$ 定义为尺度 $t\left(t=t_{1}, t_{2}, \cdots, t_{J}\right)$ 对应的谱图 小波算子, $J$ 为分解层数。则对应尺度 $t$ 的谱图小 波定义为

$$
\psi_{t, n}(m)=\sum_{l=0}^{N-1} g\left(t \lambda_{l}\right) x_{l}^{*}(n) x_{l}(m)
$$

在 SGWT 中, 尺度函数作为一个低通滤波器, 用于提取信号的低频成分。 $h$ 表示尺度函数核, 尺 度函数算子定义为 $T_{h}=h(\boldsymbol{L})$, 则尺度函数可确定为

$$
\phi_{n}(m)=\sum_{l=0}^{N-1} h\left(\lambda_{l}\right) x_{l}^{*}(n) x_{l}(m)
$$

通过求解给定图信号 $f$ 与谱图小波、尺度函数 的内积得到谱图小波系数 $W_{f}$ 和尺度系数 $S_{f}$ 分别为

$$
\begin{gathered}
W_{f}(t, n)=\left\langle\psi_{t, n}, f\right\rangle=\sum_{l=0}^{N-1} g\left(t \lambda_{l}\right) \hat{f}(l) x_{l}(n) \\
S_{f}(n)=\left\langle\phi_{n}, f\right\rangle=\sum_{l=0}^{N-1} h\left(\lambda_{l}\right) \hat{f}(l) x_{l}(n)
\end{gathered}
$$

式中, $\hat{f}(l)$ 为图信号 $f$ 的傅里叶变换。

\section{2 谱图小波阈值降噪方法}

\section{1 基于谱图理论的一维数字信号表示}

考虑如图 $2 \mathrm{a}$ 所示的一维数字信号 $f(t)$, 图 $2 \mathrm{~b}$ 为图 $2 \mathrm{a}$ 的局部放大图, 其中圆点表示一维数字信号
时间序列点的函数值, 圆点与圆点之间依次通过直 线连接, 显然一维数字信号具有如图 1 所示的路图 结构。因此, 可将一维数字信号定义到路图上, 称 为路图信号, 具体实现为: 一维数字信号的时序性 匹配路图的序列结构, 一维数字信号时间序列点的 函数值匹配路图节点的函数值。

利用 Gauss 权值函数定义路图信号相邻节点间边 的权值, 然后基于谱图理论构建路图信号的邻接矩阵 $\boldsymbol{W}$ 、度对角矩阵 $\boldsymbol{D}$ 及拉普拉斯矩阵 $\boldsymbol{L}$, 从而将一维数 字信号的相关信息保存到路图信号的矩阵中。路图信 号结构较简单, 其邻接矩阵 $\boldsymbol{W}$ 和拉普拉斯矩阵 $\boldsymbol{L}$ 的 形式也较简单, 其中邻接矩阵 $\boldsymbol{W}$ 只有次对角元素有 值, 主对角元素和其余元素全为 0 ; 拉普拉斯矩阵 $\boldsymbol{L}$ 只有主对角元素和次对角元素有值, 其余元素全为 0 。

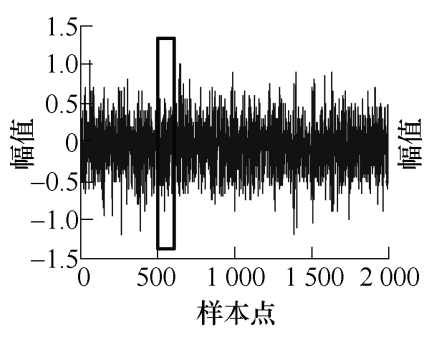

(a) 一维数字信号

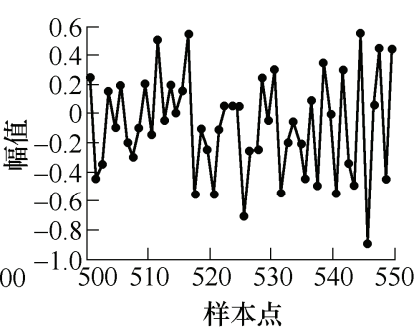

(b) 局部放大图
图 2 一维数字信号及其局部放大图

\section{2 谱图小波核与尺度函数核定义}

谱图小波核 $g$ 是原点附近的一个一元幕, 并且 随 $x$ 的增大存在幕律衰减, 满足 $g(0)=0$ 和 $\lim g(x)=0$ 。选取三次样条谱图小波核构建谱图小 波变换, 定义为

$$
g(x)= \begin{cases}x^{2} & 0 \leqslant x<1 \\ x^{3}-6 x^{2}+11 x-5 & 1 \leqslant x \leqslant 2 \\ 4 x^{-2} & x>2\end{cases}
$$

则在尺度 $t$ 下, 谱图小波算子定义为

$$
T_{g}^{t}=g(t x) \quad t=t_{1}, t_{2}, \cdots, t_{J}
$$

尺度 $t$ 由拉普拉斯特征值上界 $\lambda_{\text {max }}$ 、谱图小波变 换设计参数 $K$ 和分解层数 $J$ 共同确定。拉普拉斯特征 值下界设置为 $\lambda_{\text {min }}=\lambda_{\text {max }} / K$, 则最大尺度设置为 $t_{1}=2 / \lambda_{\text {min }}$, 最小尺度设置为 $t_{J}=1 / \lambda_{\text {max }}$, 且 $t_{1}, t_{2}, \cdots, t_{J}$ 满足对数等差分布。

谱图小波核 $g$ 随尺度 $t$ 的变化趋势如图 3 所示。 显然, $g$ 表现为带通滤波器性质, 且尺度越大, 带 宽越小, 对应子带频率越低; 反之, 带宽越大, 对 应子带频率越高。因此, 通过尺度的变换从而将路 图信号分解成不同频率区间的子带。

尺度函数核 $h$ 由拉普拉斯特征值上界 $\lambda_{\text {max }}$ 和参 数 $K$ 确定, 满足 $h(0)>0$ 和 $\lim _{x \rightarrow \infty} h(x)=0$, 定义为 


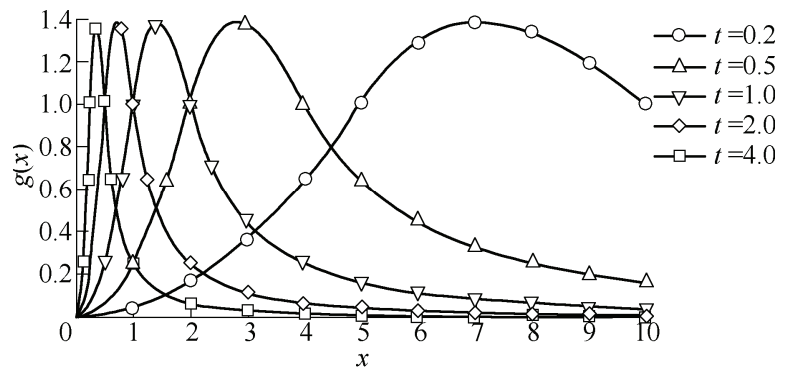

图 3 谱图小波核 $g$ 随尺度 $t$ 的变化趋势

$$
h(x)=\alpha \exp \left(-(\beta x)^{4}\right)
$$

式中, $\beta=K /\left(0.6 \lambda_{\text {max }}\right)$, 通常取 $K=20$; 设置 $\alpha$ 是 为了使 $h(0)$ 等于谱图小波核 $g$ 的最大值。

尺度函数核 $h$ 随 $\beta$ 的变化趋势如图 4 所示。由 图可知, $h$ 表现为低通滤波器性质, 在 $g$ 确定时, 其带宽只取决于路图信号的拉普拉斯特征值上界 $\lambda_{\text {max }}$ 。 $\lambda_{\text {max }}$ 越大, 带宽越大, 则对应尺度系数包含 的频率成分越多; 反之, 带宽越小, 对应尺度系数 包含的频率成分越少; 对于不同路图信号, $h$ 根据 其拉普拉斯特征值上界 $\lambda_{\text {max }}$ 产生相应变化, 具有自 适应性。

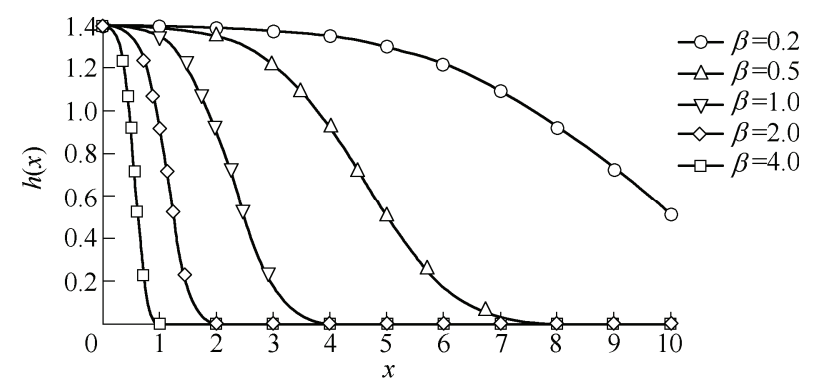

图 4 尺度函数核 $h$ 随 $\beta$ 的变化趋势

\section{3 阈值及阈值函数选取}

常用的阈值主要包括 sqtwolog、heursure、 minimaxi、rigrsure 等四种。其中, 前两种阈值降噪 较彻底, 但易把有用信号视为噪声而去除; 后两种 阈值对于存在较少高频信息的信号, 降噪效果较好, 可以将微弱的信号提取出来。针对特定信号, 应先从 理论上分析信号分布的频率范围, 从而选择合适的國 值。本文涉及的滚刀主轴振动信号主要分布在低频范 围, 可选用 minimaxi ${ }^{[19]}$ 计算阈值 $\kappa$, 表达式为

$$
\kappa= \begin{cases}\sigma_{j}(0.3936+0.1829 \times \ln N / \ln 2) & N>32 \\ 0 & N \leqslant 32\end{cases}
$$

式中, $N$ 为尺度 $t_{j}$ 对应子带的谱图小波系数个数, $\sigma_{j}$ 表示第 $j$ 子带所含噪声标准差, 由下式估计

$$
\sigma_{j}=\operatorname{median}\left(\left|d_{j}(k)\right|\right) / 0.6745
$$

式中, $d_{j}(k)$ 表示第 $j$ 子带的第 $k$ 个谱图小波系数, 分母 0.6745 表示正常的数据分布。
不同阈值函数体现了对谱图小波系数的不同处 理策略, 典型的阈值函数为硬阈值函数和软阈值函 数, 见式(18)、(19), 示意图如图 5 所示。

硬阈值函数

$$
\bar{w}_{j k}= \begin{cases}w_{j k} & \left|w_{j k}\right| \geqslant \kappa \\ 0 & \left|w_{j k}\right|<\kappa\end{cases}
$$

软阈值函数

$$
\bar{w}_{j k}= \begin{cases}{\left[\operatorname{sgn}\left(w_{j k}\right)\right]\left(\left|w_{j k}\right|-\kappa\right)} & \left|w_{j k}\right| \geqslant \kappa \\ 0 & \left|w_{j k}\right|<\kappa\end{cases}
$$

式中, $w_{j k}$ 为原始谱图小波系数, $\bar{w}_{j k}$ 为阈值函数处 理后的谱图小波系数。

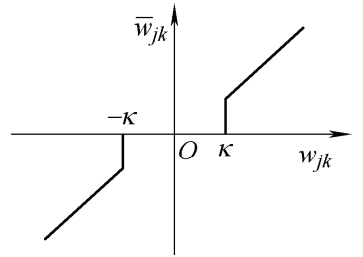

(a) 硬阈值函数

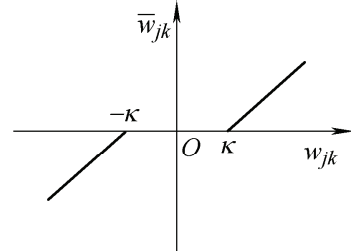

(b) 软阈值函数
图 5 典型的阈值函数

由图 5 可知, 软阈值函数是连续函数, 没有跳 跃点, 通过其处理后的谱图小波系数整体连续性更 好，重构信号不会产生附加振荡，具有原始信号的 平滑性。因此，文中选用软阈值函数。

\section{4 谱图小波阈值降噪流程}

谱图小波阈值降噪流程如图 6 所示，具体如下 所述。

(1) 将一维数字信号定义到路图上, 形成路图 信号, 再构造无向加权图 $G$, 利用权值函数建立邻 接矩阵 $\boldsymbol{W}$ 和度对角矩阵 $\boldsymbol{D}$, 计算拉普拉斯矩阵 $\boldsymbol{L}$, 并求取拉普拉斯特征值 $\lambda_{l}$ 和拉普拉斯特征矢量 $\boldsymbol{x}_{l}$ 。

(2) 设置分解层数, 结合谱图小波核和尺度函 数核, 利用定义在拉普拉斯矩阵 $\boldsymbol{L}$ 上的 SGWT 对路 图信号进行谱图小波变换, 得到尺度系数 $S_{f}$ 和谱图 小波系数 $W_{f}$ 。

（3）保留低通频率的所有尺度系数, 计算谱图 小波系数中每个子带对应的阈值 $\sigma_{j}$, 结合阈值函数 对谱图小波系数进行过滤处理, 具体为当 $\left|w_{j k}\right|$ 大于 等于 $\sigma_{j}$ 时, 则采用阈值函数以保留或收缩的方式对 其进行处理, 反之, 则令其等于 0 。

(4) 对步骤(3)处理后的尺度系数和谱图小波系 数进行谱图小波逆变换, 得到降噪信号。

结合公式(11)、(12)与(2)可知, 本方法首先利用 尺度函数提取信号的低频信息并保存到尺度系数 中, 然后利用阈值对包含了较少低频信息和全部高 频信息的谱图小波系数进行过滤处理。其中，谱图 


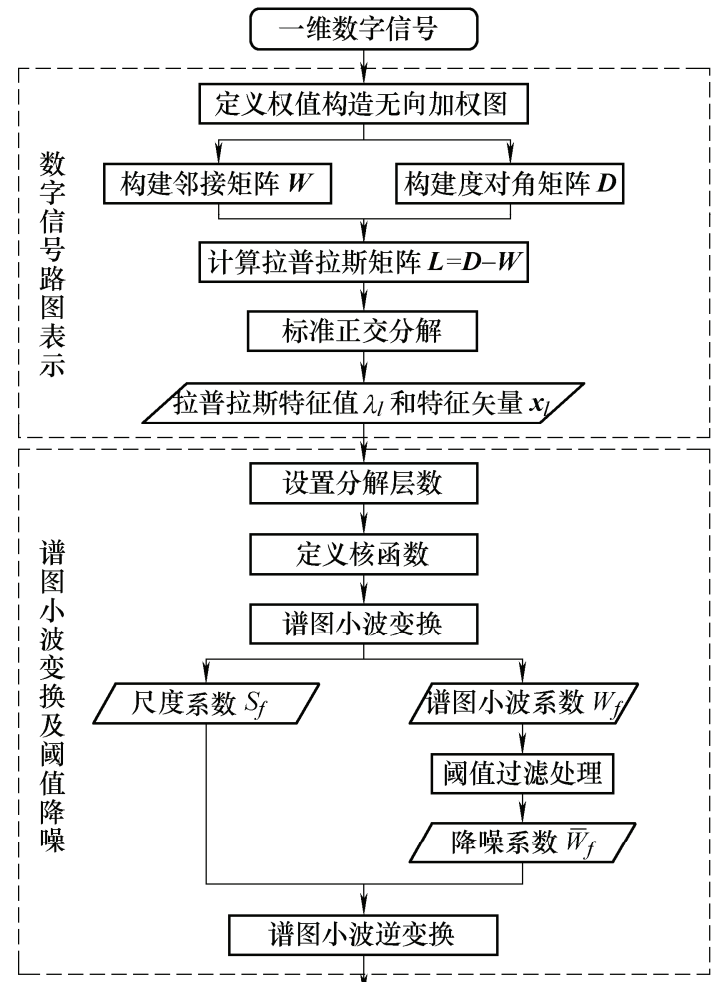

降噪信号

图 6 谱图小波阈值降噪算法流程图

小波系数中有用低频信息含量较少, 噪声占主导地 位, 利用式(17)估计的噪声标准差准确度高, 阈值的 计算更加合理, 从而实现噪声的有效去除。然而, 对 于经典小波变换, 全部有用信息和噪声混合分布于小 波系数中。进行阈值过滤时, 噪声标准差的估计值会 受到两者的相互影响。对于高频小波系数子带, 噪声 占主导, 噪声标准差估计较准确; 而对于低频小波系 数子带, 有用信息占主导, 噪声标准差的估计值准确 度低。当噪声标准差偏大时, 将造成大量有用信息被 去除; 反之, 会导致降噪不足。因此, 相比经典小波 阈值降噪方法, 本文提出方法具有一定的理论优越 性。此外, 本方法直接在谱图域内对谱图小波系数进 行一次性过滤处理, 不需迭代, 计算效率高。

\section{3 典型仿真信号降噪试验}

首先采用仿真试验验证谱图小波阈值降噪方法 的可行性和有效性, 具体包括: 进行 4 种经典仿真 信号 Heavy sine、Doppler、Blocks、Bumps 的降噪 试验, 分析不同分解层数对降噪性能的影响, 比较 该方法与经典小波阈值降噪方法的降噪性能。

\section{1 降噪性能评价指标}

降噪方法的性能有两种评价方式 ${ }^{[20]}$ : (1) 对于 通过叠加高斯白噪声形成的人工噪声信号, 其原始 清洁信号已知, 可采用原始清洁信号、降噪信号和
噪声作为性能评价参数; (2) 对于实际的噪声信号, 原始清洁信号未知, 则只能使用原始噪声信号、降 噪信号和噪声作为性能评价参数。本文采用第一种 方式评价经典仿真信号降噪试验中的降噪性能。

常用的降噪性能评价指标包括信噪比(Signal to noise ratio, SNR)、均方根差百分比(Percentage root mean square difference, PRMSD)、相关系数 (Correlation coefficient, CC)以及均方根误差(Mean square error, MSE)等, 本文采用信噪比 $(S N R)$ 评价 指标, 便于与仿真信号输入信噪比形成直观对比。 信噪比 $(S N R)$ 定义为 ${ }^{[21]}$

$$
S N R=10 \lg \left(\sum_{i=1}^{N} x(i)^{2} / \sum_{i=1}^{N}[x(i)-\tilde{x}(i)]^{2}\right)
$$

式中, $x(i)$ 表示原始清洁信号, $\tilde{x}(i)$ 表示降噪信号; 对于降噪方法来说, 信噪比 $S N R$ 越大, 降噪性能越好。

\section{2 仿真信号降噪试验过程}

设定仿真信号数据长度为 2048 , 分解层数为 3 , 通过叠加高斯白噪声设定输入信噪比 $S N R_{\text {in }}=$ $10 \mathrm{~dB}$ 。4 种仿真信号降噪过程图如表 1 所示, 包括 原始清洁信号、噪声信号、降噪前系数、降噪后系 数和降噪信号。

由表 1 可知, 降噪前系数中, $D_{0}$ 表示尺度系数, 反映噪声信号中的低频成分, 其构成的曲线较平滑、 波动小，与原始清洁信号形状相近; $D_{1} 、 D_{2} 、 D_{3}$ 分 别为尺度 $t\left(t=\left\{t_{j}\right\}_{j=1}^{3}\right)$ 对应的谱图小波系数, 显然有 效信号能量集中在谱图域中的一些较大的系数中, 且从 $D_{1}$ 到 $D_{3}$ 波动逐渐增大, 对应原始清洁信号特 征逐渐减弱。降噪后系数中, 较大的谱图小波系数 得到了保留, 大部分较小的谱图小波系数减小至零。 四种降噪信号都较平滑, 与原始清洁信号接近, 输 出信噪比 $S N R_{\text {out }}$ 较之输入信噪比 $S N R_{\text {in }}$ 有很大提 升。仿真结果验证了本方法可用于一维数字信号降 噪, 且降噪效果明显。

根据仿真试验结果分析可得：谱图小波变换具 有较强的去数据相关性能力, 能够使信号的能量集 中于谱图域子带较大的谱图小波系数中, 而噪声的 能量分布于整个谱图域子带内。即在谱图域各子带 内, 幅值较大的谱图小波系数一般以信号为主，幅 值较小的谱图小波系数很大程度上是噪声。通过设 定阈值可以保留信号对应的谱图小波系数, 将绝大 部分噪声系数减小至零。

\section{3 不同分解层数降噪性能对比}

设定 4 种经典仿真信号 Heavy sine、Doppler、 Blocks、Bumps 的 $S N R_{\text {in }}$ 从 $0 \mathrm{~dB}$ 递增至 $20 \mathrm{~dB}$, 分 别进行 1 至 10 层谱图小波变换, 降噪结果如图 7 所示。 
表 1 四种仿真信号降噪试验过程

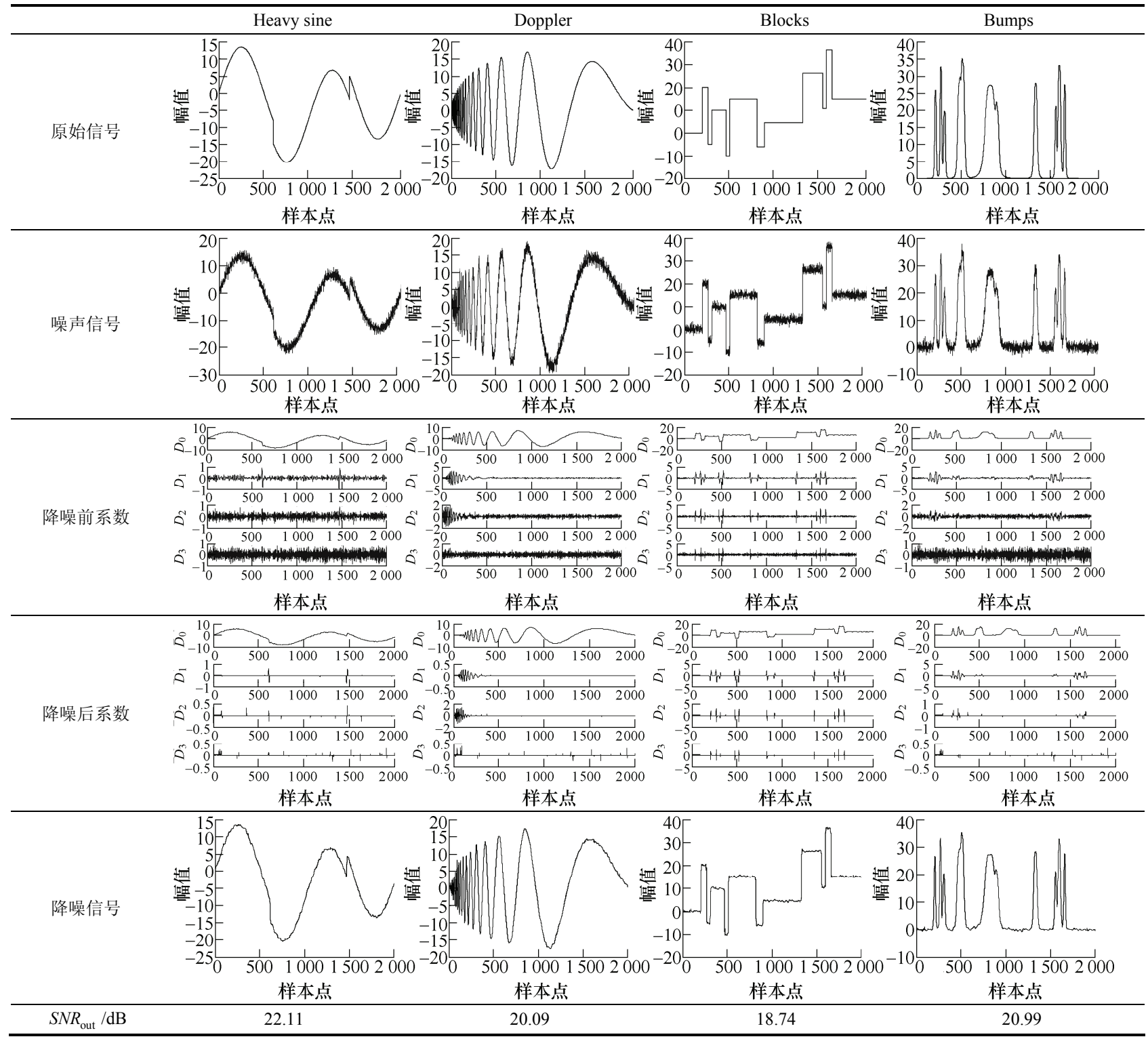

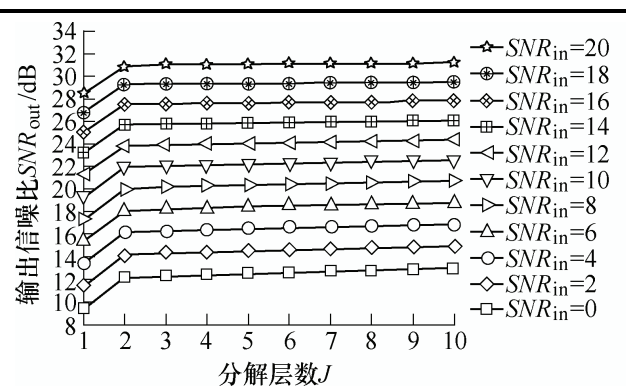

(a) 仿真信号Heavy sine

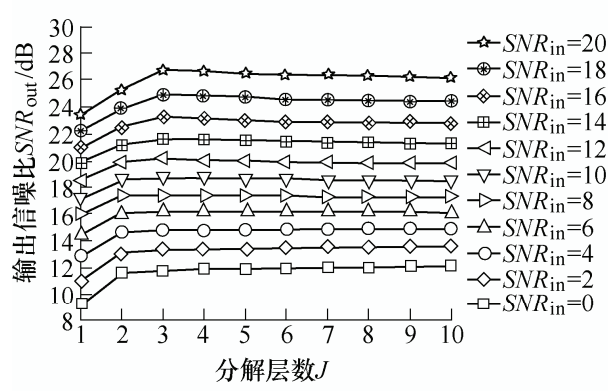

(c) 仿真信号Blocks

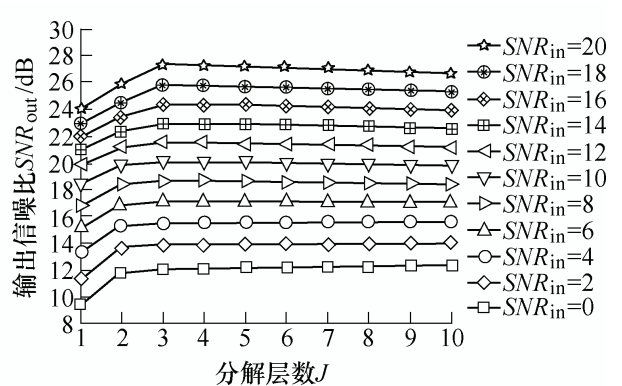

(b) 仿真信号Doppler

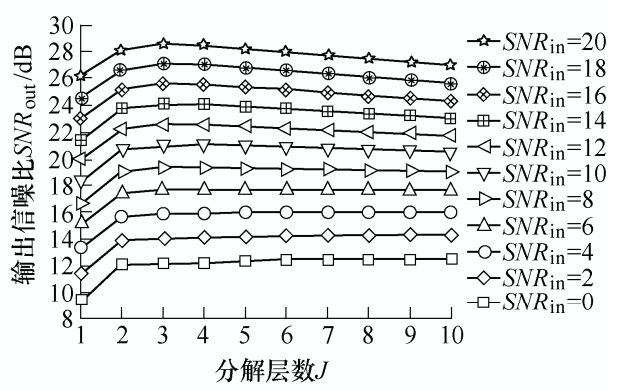

(d) 仿真信号Bumps

图 7 不同分解层数降噪性能对比 
从图 7 可知, 对于 Heavy sine 信号, 不同 $S N R_{\mathrm{in}}$ 下, $S N R_{\text {out }}$ 随分解层数的增大而逐渐增大, 变化趋 势相同, 但从 $2 \sim 10$ 层 $S N R_{\text {out }}$ 变化平稳, 降噪性能 提升不明显。对于 Doppler、Blocks、Bumps 信号, 当 $S N R_{\text {in }}$ 较小 $(0 \sim 8 \mathrm{~dB})$ 时, $S N R_{\text {out }}$ 随分解层数的增 大而逐渐增大, 从 $2 \sim 10$ 层 $S N R_{\text {out }}$ 变化也平稳; 当 $S N R_{\text {in }}$ 较大 $(9 \sim 20 \mathrm{~dB})$ 时, $S N R_{\text {out }}$ 随分解层数的增 大而先增大后缓慢减小, 在分解层数为 3 时达到 最大, 但从 $4 \sim 10$ 层 $S N R_{\text {out }}$ 变化也较平稳, 也能 获得较好的降噪性能。经综合分析, 分解层数选 取 3 6 层可保证较好的降噪性能; 3 层为最佳分 解层数, 不仅能获得较好的降噪性能, 还能保证 较高的计算效率。

\section{4 与经典小波阈值降噪方法性能对比}

采用文献[22]所用的单因素分析法在四种经典 仿真信号上验证了经典小波阈值降噪方法中, “ $\mathrm{db} 7$ ”、“sym5” 和 “coif1” 小波基结合软阈值函数
可以达到较高的降噪性能。将提出方法与上述三种 经典小波阈值降噪方法进行对比试验。设定 4 种经 典仿真信号 Heavy sine、Doppler、Blocks、Bumps 的 $S N R_{\mathrm{in}}$ 从 $0 \mathrm{~dB}$ 递增至 $20 \mathrm{~dB}$, 四种方法同时进行 3 层分解。表 2 所示为 $S N R_{\mathrm{in}}=10 \mathrm{~dB}$ 时, 四种方法 的降噪信号时域图。显然, 本方法对应的降噪信号 平滑度更高、波动更小, 与原始清洁信号更相似， 且降噪信号的信噪比更大。经典小波阈值降噪方法 对应的降噪信号平滑度较低、波动较大, 其中, “db7” 和 “sym5” 小波基的降噪性能相近, 稍优于 “coif1” 小波基。上述表明本方法降噪性能优于经典 小波阈值降噪方法。

图 8 所示为 4 种经典仿真信号输出信噪比 $S N R_{\text {out }}$ 随输入信噪比 $S N R_{\text {in }}$ 的变化趋势。图示表明 $S N R_{\text {in }}$ 在 $0 \sim 20 \mathrm{~dB}$ 区间范围内, 本文提出方法的降 噪性能比经典小波阈值降噪方法的降噪性能高, 进 一步说明了本方法的有效性。

表 2 四种方法降噪信号时域图

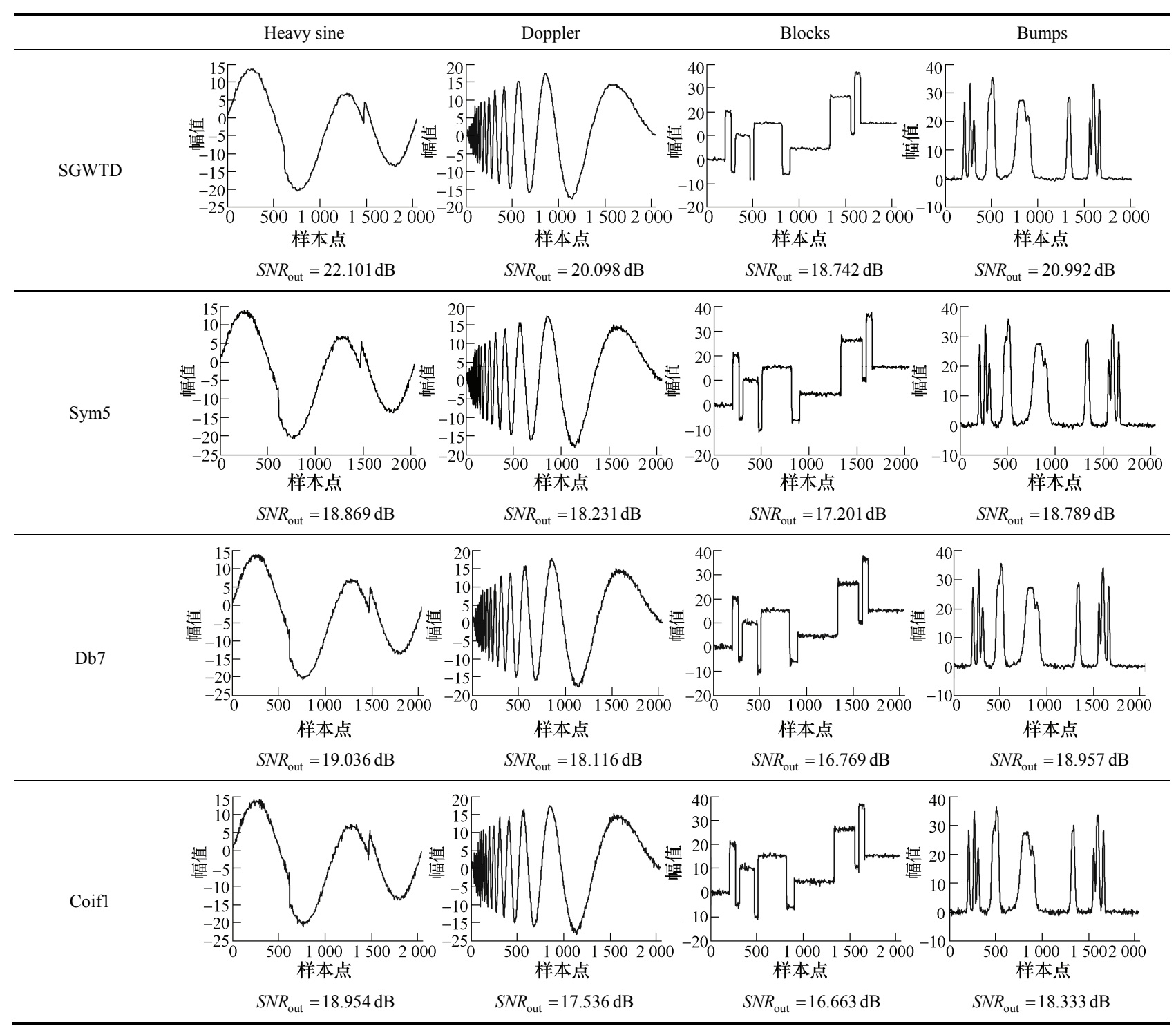




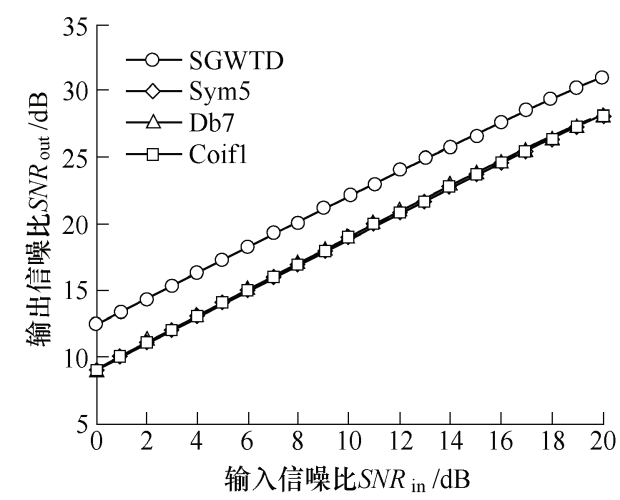

(a) 仿真信号Heavy sine

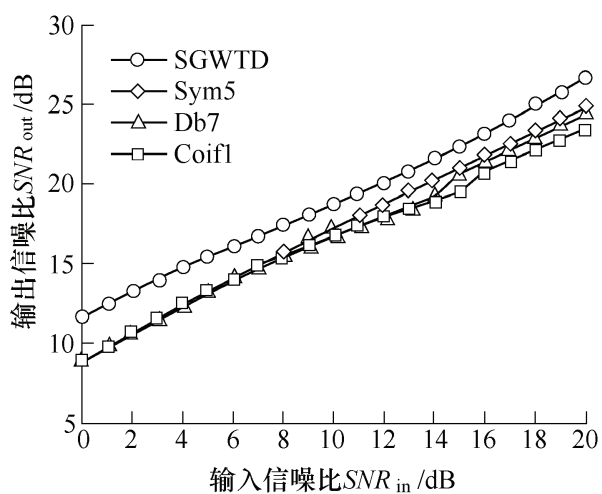

(c) 仿真信号Blocks

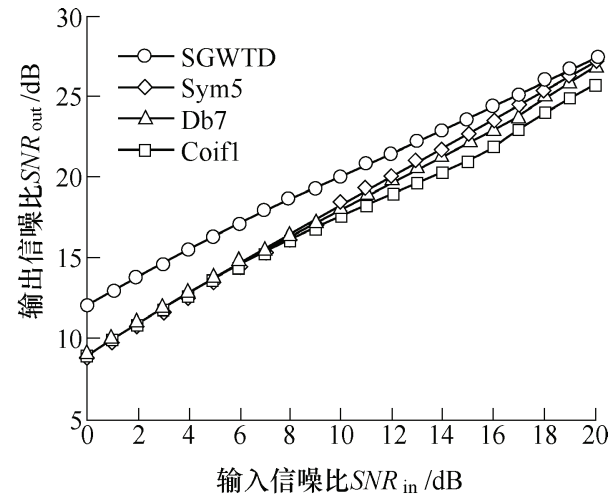

(b) 仿真信号Doppler

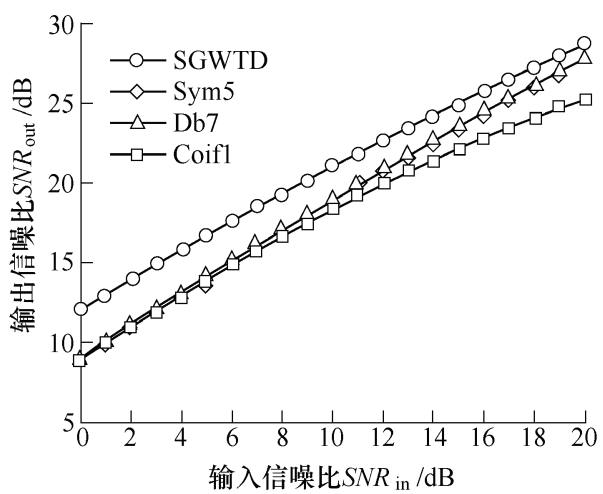

(d) 仿真信号Bumps

图 8 四种方法输出信噪比随输入信噪比的变化趋势

\section{4 工程应用}

\section{1 滚刀主轴振动信号采集}

为了验证本文提出方法对实际工程信号降噪的 有效性, 在某汽车传动件生产企业进行滚齿机加工 过程中滚刀主轴振动信号的采集试验。试验中, 滚 齿机加工信息如表 3 和表 4 所示, 振动信号采集系 统仪器布置如图 9 所示。PCB 加速度传感器安装 在滚刀主轴轴承支座上, 采集 $z$ 向振动, 采样频率 为 $10000 \mathrm{~Hz}$ 。振动信号经加速度传感器、信号调 理仪保存到存储记录仪中, 并显示振动信号时域波 形。滚齿加工为断续切削过程 ${ }^{[23]}$, 因此非线性、非 平稳的滚刀主轴振动信号呈明显的周期交替性变 化, 其样本如图 10 所示。

表 3 工件基本参数

\begin{tabular}{cc}
\hline 参数 & 数值 \\
\hline 齿数 $z_{g}$ & 29 \\
模数 $m_{g} / \mathrm{mm}$ & 3.5 \\
压力角 $\alpha /\left(^{\circ}\right)$ & 20 \\
螺旋角 $\beta /\left(^{\circ}\right)$ & 0 \\
齿宽 $b / \mathrm{mm}$ & 13 \\
\hline
\end{tabular}

表 4 滚刀基本参数

\begin{tabular}{cc}
\hline 参数 & 数值 \\
\hline 头数 $z_{h}$ & 2 \\
模数 $m_{h} / \mathrm{mm}$ & 3.5 \\
槽数 $z_{k}$ & 14 \\
转速 $n /(\mathrm{r} / \mathrm{min})$ & 680 \\
进给速度 $f_{s} /(\mathrm{mm} / \mathrm{min})$ & 55 \\
\hline
\end{tabular}

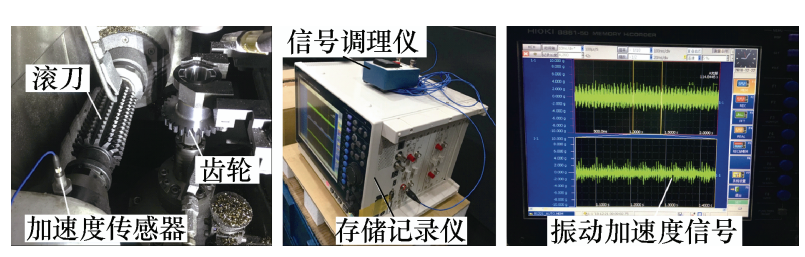

图 9 振动信号采集系统仪器布置

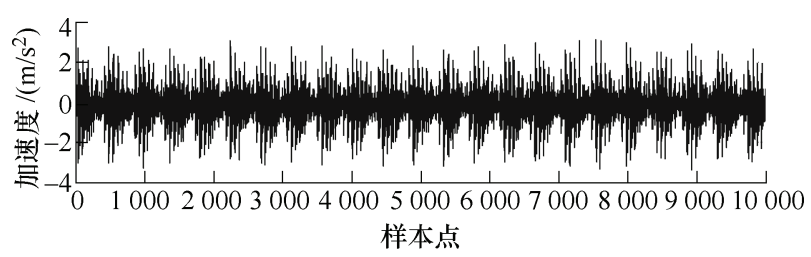

图 10 滚刀主轴振动信号

\section{2 滚刀主轴振动信号包络谱分析}

包络谱分析是通过对信号进行 Hilbert 变换后 再进行 FFT 变换, 从而得到包络谱, 其更易从复杂 
的信号中突显出有用信息 ${ }^{[24]}$ 。滚刀主轴振动信号包 络谱中主要的有用频率包括滚刀主轴自转频率和滚 切频率 ${ }^{[23]}$ 。

滚刀主轴自转频率定义为

$$
f_{r}=n / 60
$$

式中, $n$ 为滚刀主轴转速。由此可得主轴自转频率 及其倍频如表 5 所示。

表 5 主轴自转频率及其倍频

\begin{tabular}{ccccc}
\hline 倍数 & 1 & 2 & 3 & 4 \\
\hline 频率 $f_{r} / \mathrm{Hz}$ & 11.33 & 22.66 & 33.99 & 45.32 \\
\hline \hline 倍数 & 5 & 6 & 7 & 8 \\
\hline 频率 $f_{r} / \mathrm{Hz}$ & 56.65 & 67.98 & 79.31 & 90.64 \\
\hline
\end{tabular}

滚切频率定义为

$$
f_{g}=n z_{k} / 60
$$

式中, $n$ 为滚刀主轴转速, $z_{k}$ 表示滚刀槽数。滚切 频率及其倍频如表 6 所示。

表 6 滚切频率及其倍频

\begin{tabular}{ccccc}
\hline 倍数 & 1 & 2 & 3 & 4 \\
\hline 频率 $f_{g} / \mathrm{Hz}$ & 158.67 & 317.34 & 476.01 & 634.68 \\
\hline \hline 倍数 & 5 & 6 & 7 & 8 \\
\hline 频率 $f_{g} / \mathrm{Hz}$ & 793.35 & 952.08 & 1110.69 & 1269.36 \\
\hline
\end{tabular}

表 5、6 的理论分析结果表明滚刀主轴振动信号 中的有用频率成分都集中在 $1500 \mathrm{~Hz}$ 以内的低频 段。对实测滚刀主轴振动信号进行包络谱分析, 包 络谱及其低频段详细信息分别如图 11 和 12 所示。

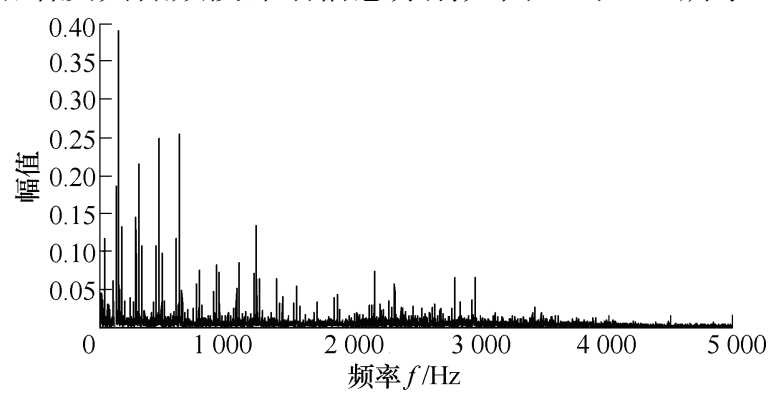

图 11 滚刀主轴振动信号包络谱

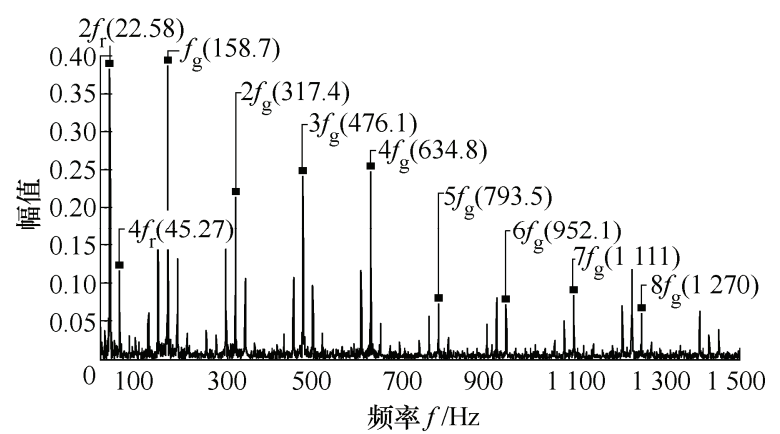

图 12 滚刀主轴振动信号包络谱低频段特征
比较图 12 与表 5、6 中数据可知, 实测滚刀主轴振 动信号频率特征与理论分析数据吻合，说明大于 $1500 \mathrm{~Hz}$ 的高频段主要为噪声, 其主要分布在 $1500 \sim 4000 \mathrm{~Hz}$ 频率段。因此, 降噪实际是消除大 于 $1500 \mathrm{~Hz}$ 的高频段信号。

\section{3 滚刀主轴振动信号谱图小波阈值降噪}

对滚刀主轴振动信号进行 3 层谱图小波变换, 并利用阈值对谱图小波系数进行过滤，结果如图 13 所示。图 $13 \mathrm{a}$ 为过滤前的尺度系数和谱图小波系数, 观察可知从 $D_{1}$ 到 $D_{3}$ 谱图小波系数周期性明显减弱, 混乱程度增大, 说明有效信号占比越来越小，与仿 真结果相同。基于滚齿加工的断续切削特性，滚刀 主轴振动信号相邻周期之间的信号主要为噪声。 图 $13 \mathrm{~b}$ 中显示阈值函数处理后的系数保留了部分周 期之中的系数，周期之间的系数大部分减小至 0 , 表明噪声信号得到了有效过滤。

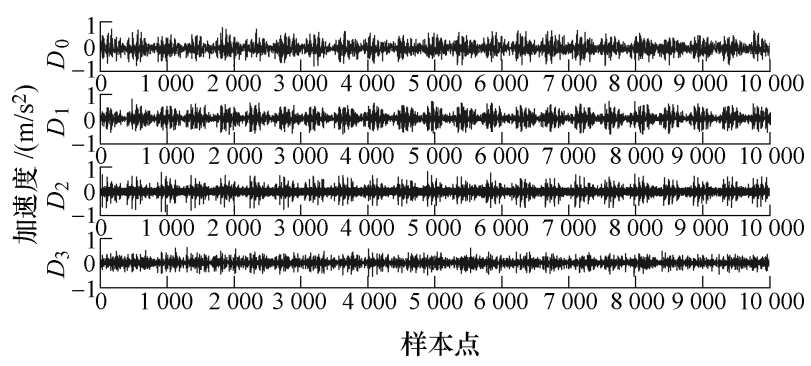

(a) 尺度系数与谱图小波系数

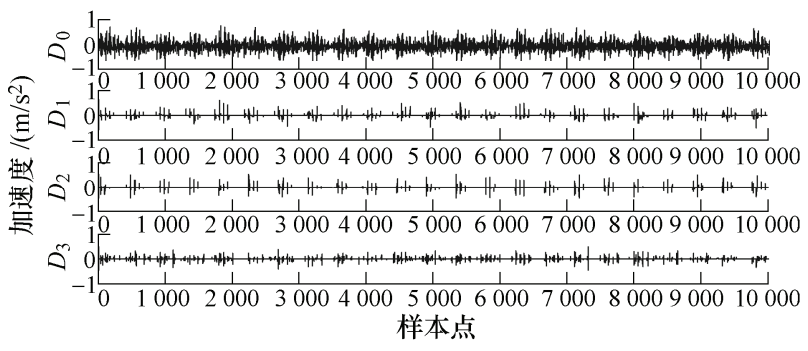

(b) 尺度系数与降噪谱图小波系数

图 13 振动信号谱图小波阈值降噪过程

图 $14 \mathrm{a} \sim 14 \mathrm{~d}$ 所示分别为本文提出方法与三种 经典小波阈值降噪方法的降噪信号时域图。通过比 较图 14a 与图 $14 \mathrm{~b} \sim 14 \mathrm{~d}$ 可知, SGWTD 降噪信号周 期之间的过渡信号明显减弱，相比于原始信号周期 性加强, 更加突显出滚齿加工的断续切削过程。此 外, 加工过程中切削参数保持不变, 滚刀切削齿轮 时所受到的周期性交变冲击载荷基本恒定, 其产生 的振动响应变化较平稳, 可从图 10 所示的原始主轴 振动信号中看出。经降噪处理后, SGWTD 降噪信 号的幅值变化平稳, 与原始信号相似; 而 Sym5、 Db7 和 Coif1 降噪信号的幅值波动大, 其损失了部 分时域信息, 可见图 $14 b \sim 14 d$ 中椭圆标注位置。 SGWTD 降噪信号信噪比为 $4.027 \mathrm{~dB}$, 大于 Sym5、 
Db7 和 Coif1 降噪信号。综合上述分析, 本文提出 方法能够有效消减滚刀主轴振动信号中的噪声, 明显 优于图 $14 \mathrm{~b} \sim 14 \mathrm{~d}$ 所示的三种经典小波阈值降噪方法。

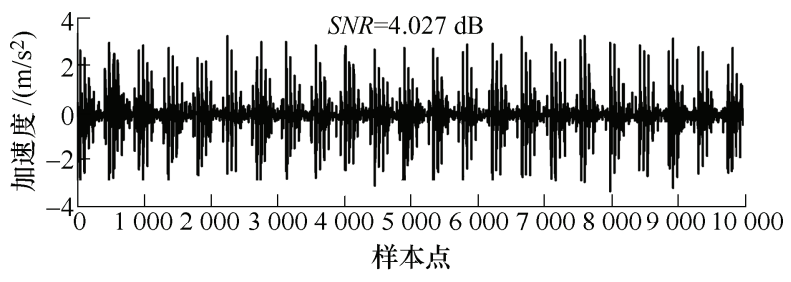

(a) SGWTD 降噪信号

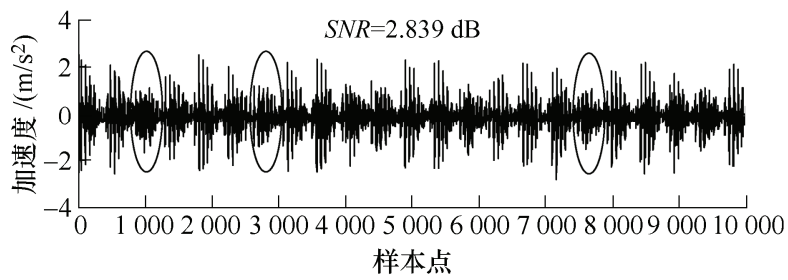

(b) Sym5 降噪信号

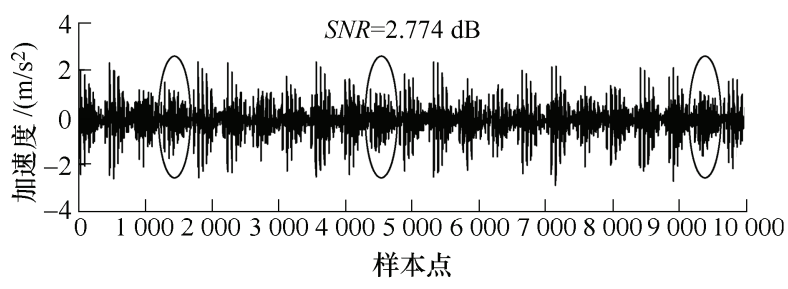

(c) Dd7 降噪信号

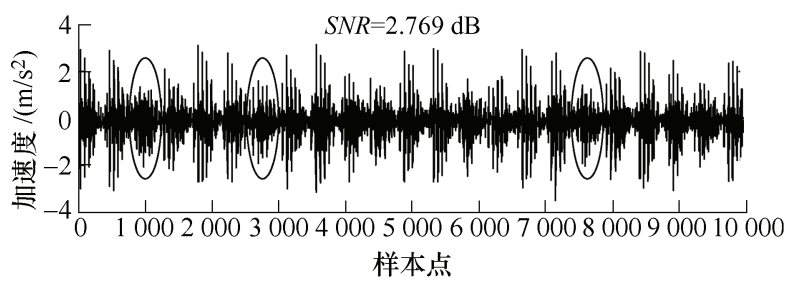

(d) Coif1 降噪信号

图 14 四种方法降噪信号

对图 14 中降噪信号进行包络谱分析, 分别如 图 15a 15d 所示。图 15a 所示的 SGWTD 降噪信号 包络谱中, 大于 $1500 \mathrm{~Hz}$ 的高频段基本得到消除, 剩余频率主要为主轴自转频率、滚切频率等有用成 分, 且主轴自转频率特征 $\left(2 f_{r} 、 4 f_{r} 、 6 f_{r} 、 8 f_{r}\right)$ ) 突出, 滚切频率特征 $\left(f_{g} 、 2 f_{g} 、 3 f_{g} 、 4 f_{g} 、 5 f_{g} 、 6 f_{g}\right)$ 中心频率 明显, 周边干扰成分少。而图 $15 \mathrm{~b} \sim 15 \mathrm{~d}$ 所示的 Sym5、Db7 和 Coif1 降噪信号包络谱中, 存在大于

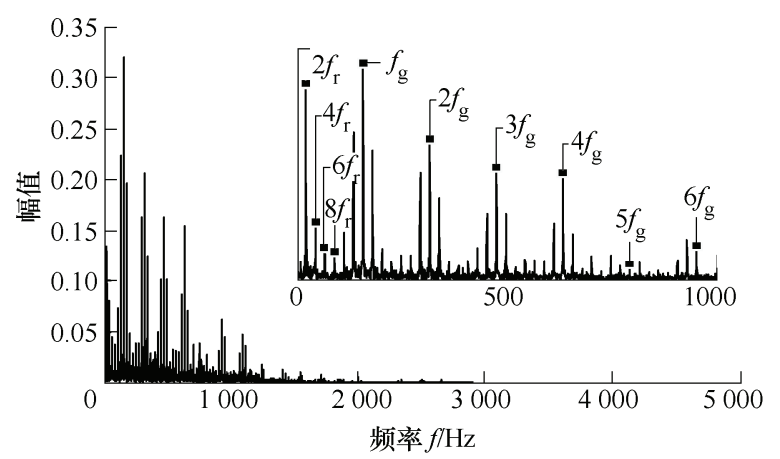

(a) SGWTD 降噪信号包络谱

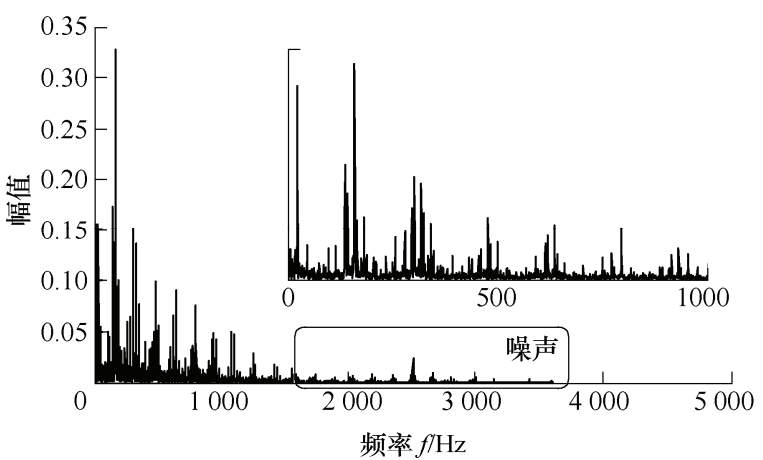

(b) Sym5 降噪信号包络谱

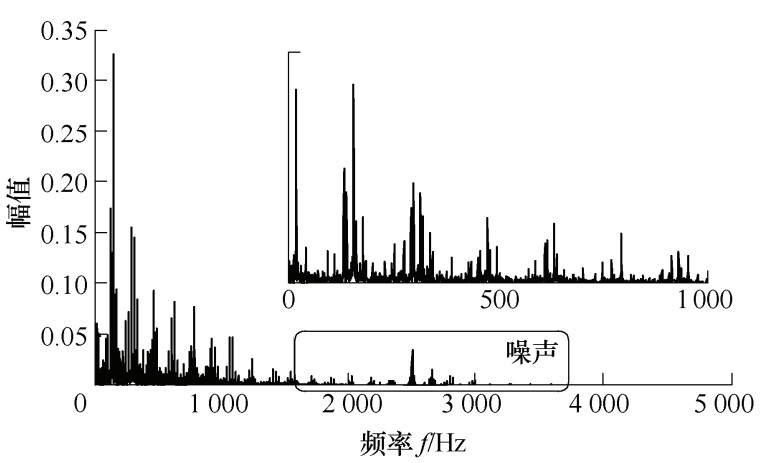

(c) Dd7 降噪信号包络谱

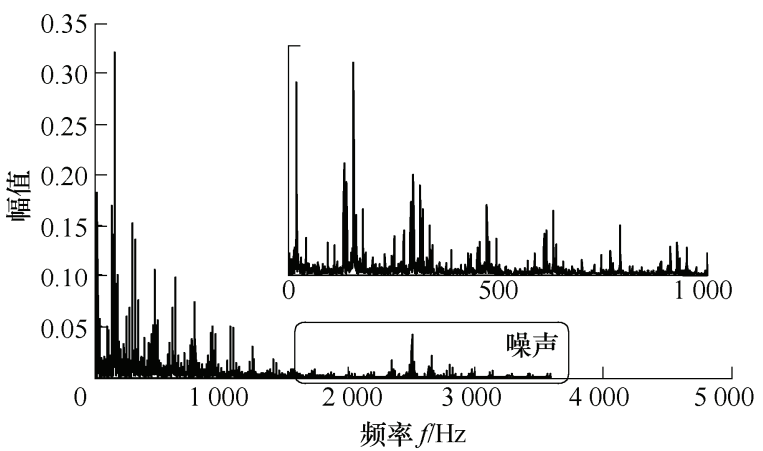

(d) Coif1 降噪信号包络谱

图 15 四种方法降噪信号包络谱图

$1500 \mathrm{~Hz}$ 的噪声频率, 主要集中在 $1500 \sim 3500 \mathrm{~Hz}$ 频率段, 且主轴自转频率特征不明显, 滚切频率 特征周边干扰成分较多。经综合分析, 在实际工 程信号降噪应用中，本文提出方法优于经典小波 阈值降噪方法。

\section{5 结论}

(1) 参照谱图小波变换在平面图像、三维实体 分析中的应用，提出了一种新的基于谱图小波变换 的阈值降噪方法, 实现了谱图小波变换在一维数字 信号降噪中的应用。

(2) 将谱图小波阈值降噪方法用于典型仿真信 号降噪试验, 结果验证了该方法用于一维数字信号 降噪的可行性和有效性, 并对强、弱噪声情况都表 现出很高的降噪性能, 优于经典小波阈值降噪方法。 
(3) 将谱图小波阈值降噪方法用于滚刀主轴振 动信号降噪试验, 结果表明该方法可有效地消减振 动信号中的高频噪声成分, 并保留低频有用信息, 实现了机械振动信号的快速非迭代降噪。

下一步拟将所提方法应用到不同对象，并探讨 谱图小波核、分解层数、阈值及阈值函数等关键参 数的优化选择问题。

\section{参 考 文 献}

[1] 周玉清, 梅雪松, 姜歌东, 等. 基于内置传感器的大型 数控机床状态监测技术 [J]. 机械工程学报, 2009, 45(4): 125-130.

ZHOU Yuqing, MEI Xuesong, JIANG Gedong, et al. Technology on large scale numerical control machine tool condition monitoring based on built-in sensors[J]. Journal of Mechanical Engineering, 2009，45(4): 125-130.

[2] 何志坚, 周志雄, 黄向明. 基于变分模态分解的关联维 数及相关向量机的刀具磨损状态监测 $[\mathrm{J}]$. 计量学报, 2018, 39(2): 182-186.

HE Zhijian, ZHOU Zhixiong, HUANG Xiangming. Tool wear state monitoring based on variational mode decomposition and correlation dimension and relevance vector machine[J]. Acta Metrologica Sinica, 2018, 39(2): 182-186.

[3] 王丽华, 杨家巍, 张永宏, 等. 基于堆叠降噪自编码的 刀具磨损状态识别 [J]. 中国机械工程, 2018，29(17): 2038-2045.

WANG Lihua, YANG Jiawei, ZHANG Yonghong, et al. Tool wear condition recognition based on SDAE[J]. China Machinery Engineering, 2018, 29(17): 2038-2045.

[4] 王志坚, 常雪, 王俊元, 等. 排列熵优化改进变模态分 解算法诊断齿轮箱故障[J]. 农业工程学报, 2018, 34(23): 59-66.

WANG Zhijian, CHANG Xue, WANG Junyuan, et al. Gearbox fault diagnosis based on permutation entropy optimized variational mode decomposition[J]. Transactions of the Chinese Society of Agricultural Engineering, 2018, 34(23): 59-66.

[5] 刘又午, 章青, 赵小松, 等. 基于多体理论模型的加工 中心热误差补偿技术 $[\mathrm{J}]$. 机械工程学报, 2002, 38(1): 127-130.

LIU Youwu, ZHANG Qing, ZHAO Xiaosong, et al. Thermal error compensation technology of machining center based on multi-body theory model[J]. Chinese Journal of Mechanical Engineering, 2002, 38(1): 127-130.
[6] 李建卓. 匹配追踪算法在振动信号去噪中的应用 [J]. 计算机与数字工程, $2013,41(12): 1918-1919,1988$. LI Jianzhuo. Application of matching pursuit algorithm in vibration signal denoising $[\mathrm{J}]$. Computer and Digital Engineering, 2013, 41(12): 1918-1919, 1988.

[7] TAY D B H, TANAKA Y, SAKIYAMA A. Almost tight spectral graph wavelets with polynomial filters[J]. IEEE Journal of Selected Topics in Signal Processing, 2017, 11(6): $812-824$.

[8] HAMMOND D K, GRIBONVAL R. Wavelets on graphs via spectral graph theory[J]. Applied and Computational Harmonic Analysis, 2011, 30(2): 129-150.

[9] LEANDRO J D J G, JUNIOR R M C, FERIS R S. Shape analysis using the spectral graph wavelet transform[C] // IEEE International Conference on Escience. IEEE, 2013: 307-316.

[10] DONG X, ORTEGA A, FROSSARD P, et al. Inference of mobility patterns via spectral graph wavelets[C] // IEEE International Conference on Acoustics, IEEE, 2013: 3118-3122.

[11] OZDEMIR A, AVIYENTE S. Graph wavelet transform: Application to image segmentation[C]// Conference on Signals, Systems \& Computers, IEEE, 2014: 496-499.

[12] BEHJAT H, LEONARDI N, SÖRNMO L, et al. Anatomically-adapted graph wavelets for improved group-level fMRI activation mapping[J]. NeuroImage, 2015, 123(6): 185-199.

[13] DEUTSCH S, ORTEGA A, MEDIONI G. Manifold denoising based on spectral graph wavelets[C]// IEEE International Conference on Acoustics, IEEE, 2016: 4673-4677.

[14] 王林, 宋星. 基于谱图小波变换的图像压缩编码方法 [J]. 计算机系统应用，2018，27(5): 176-180.

WANG Lin, SONG Xing. Image compression and coding method based on spectral graph wavelet transform[J]. Computer Systems \& Applications, 2018, 27(5): 176-180.

[15] MASOUMI M, LI C, HAMZA A B. A spectral graph wavelet approach for nonrigid 3D shape retrieval[J]. Pattern Recognition Letters， 2016， 83(3): 339-348.

[16] 胡玲, 汪政, 郭啸. 谱流形小波及其在三维几何处理中 的应用 [J]. 湖南师范大学自然科学学报, 2018, 41(4): $77-84$.

HU Ling, WANG Zheng, GUO Xiao. Spectral manifold wavelet with application in $3 \mathrm{D}$ geometric processing $[\mathrm{J}]$. Journal of Natural Science of Hunan Normal University, 
2018, 41(4): 77-84.

[17] 胡玲, 李钦松, 刘圣军, 等. 三维模型匹配的谱图小波 描述符[J]. 浙江大学学报, 2019, 53(4): 761-769.

HU Ling, LI Qinsong, LIU Shengjun, et al. Spectral graph wavelet descriptor for $3 \mathrm{D}$ shape matching $[\mathrm{J}]$. Journal of Zhejiang University, 2019, 53(4): 761-769.

[18] CHUNG F R K. Spectral graph theory[M]. Fresno: American Mathematical Society, 1997.

[19] KARTHIKEYAN P, MURUGAPPAN M, YAACOB S. ECG signal denoising using wavelet thresholding technique in human stress assessment[J]. International Journal on Electrical Engineering and Informatics, 2012, 4 (2): 306-319.

[20] SADOOGHI M S, KHADEM S E. A new performance evaluation scheme for jet engine vibration signal denoising $[\mathrm{J}]$. Mechanical Systems and Signal Processing, 2016, 76-77(4): 201-212.

[21] HAO H, LIU M, XIONG P, et al. Multi-lead model-based ECG signal denoising by guided filter[J]. Engineering Applications of Artificial Intelligence， 2019， 79(2): 34-44.

[22] 刘奎, 张冬梅, 于光, 等. 空气耦合超声信号的小波阈 值滤噪试验研究[J]. 机械工程学报, 2015, 51(20): 61-66.
LIU Kui, ZHANG Dongmei, YU Guang, et al. Wavelet threshold denoising experiments of air-coupled ultrasonic signal[J]. Journal of Mechanical Engineering, 2015, 51(20): 61-66.

[23] 雷涛, 曹华军, 朱利斌, 等. 交变冲击载荷下高速干切 滚刀主轴系统振动响应特性研究 $[\mathrm{J}]$. 机械工程学报, 2017, 53(11): 113-121.

LEI Tao, CAO Huajun, ZHU Libin, et al. Vibration response characteristics research for the hob spindle system of high-speed dry hobbing under alternating impact load[J]. Journal of Mechanical Engineering, 2017, 53(11): 113-121.

[24] 孙鹏. 基于频谱分析和包络谱分析的滚动轴承故障诊 断[J]. 振动与冲击, 2012, 5(31): 189-192.

SUN Peng. Rolling bearing fault diagnosis of based on spectrum and envelope spectrum analysis[J]. Journal of Vibration and Shock, 2012, 5(31): 189-192.

作者简介: 董釒, 男, 1991 年出生, 博士研究生。主要研究方向为智能 制造技术与系统, 刀具故障预测与健康管理。

E-mail: xindong0531@foxmail.com

李国龙(通信作者), 男, 1968 年出生, 博士, 教授, 博士研究生导师。 主要研究方向为智能制造技术与装备, 精密与超精密加工技术。

E-mail: glli@cqu.edu.cn 